\title{
Selected CMS Measurements of the Top Quark Properties in Single-top Production
}

\author{
Abideh Jafari ${ }^{* \dagger}$ \\ Université catholique de Louvain, Louvain-la-Neuve, BE \\ E-mail: abideh.jafari@cern.ch
}

\begin{abstract}
A selected set of CMS measurements of the top quark properies in $t$-channel single top events together with searches for anomalous top quark couplings are presented. In particular the first $\mathrm{tZq}$ cross section measurement is briefly described. All results are based on the analysis of the LHC data at $\sqrt{s}=8 \mathrm{TeV}$ with an integrated luminosity of $19.7 \mathrm{fb}^{-1}$.
\end{abstract}

38th International Conference on High Energy Physics 3-10 August 2016

Chicago, USA

${ }^{*}$ Speaker.
†On behalf of the CMS collaboration 


\section{Introduction}

The production of top quark through electroweak interaction, the single top quark production, provides the unique opportunity to study the distinctive features of the Wtb vertex looking for signs of physics beyond the standard model (SM). At the LHC, the single top quark is dominantly produced in the $t$-channel process (Fig. 1 (a)) [1,2], and to a lesser extent, in association with a W boson (Fig. 1 (b)) [3,4]. The production through $s$-channel processes (Fig. 1 (c)) has not yet been observed at the LHC $[5,6]$. At the LHC center-of-mass energies, heavy final states containing

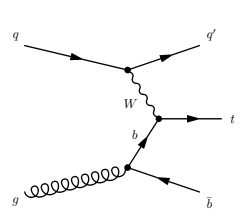

(a)

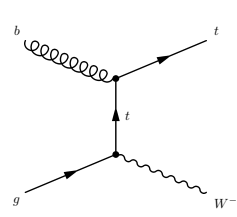

(b)

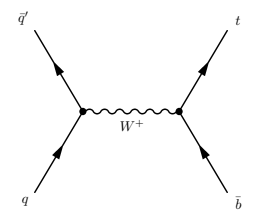

(c)

Figure 1: Representative Feynman diagrams for the production of single top quark in $t$-channel (a), in association with a $\mathrm{W}$ boson (b) and in $s$-channel (c) at leading order.

single top quark together with a $\mathrm{Z}$ boson (Fig. 2 (a)) can also be produced. Moreover, some new physics scenarios predict a relatively high probability for the $\mathrm{tZq}$ and $\mathrm{t} \gamma \mathrm{q}$ flavor-changing-neutralcurrent (FCNC) interactions (Fig. 2(b)). Compared with similar studies using tt production, the

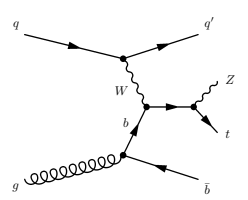

(a)

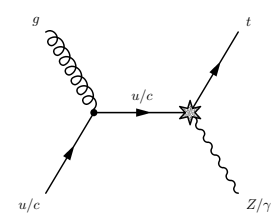

(b)

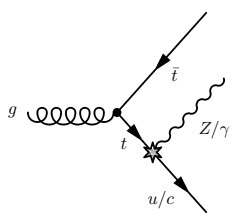

(c)

Figure 2: Representative Feynman diagrams for the production of single top quark together with $\mathrm{Z} / \gamma$ in SM (a) and as expected from FCNC (b). The FCNC process in top quark decay is shown in (c).

overwhelming top quark production at the LHC via strong interaction, single-top measurements and searches bring complementary physics information from a different production mechanism and kinematic phase space. In particular, single-top analyses are in some cases able to provide a similar or even better precision than that of $t \bar{t}$ (cf. Sec. 2).

This note presents a selected set of CMS measurements of the top quark properties and searches in the single top quark sector, all using the LHC data samples at $8 \mathrm{TeV}$ with an integrated luminosity of $19.7 \mathrm{fb}^{-1}$.

\section{Top Quark Mass Measurement}

The top quark mass is measured using $t$-channel single top events in the $\mu$ plus two jets final state where one of the jets is identified as a b jet [7]. The large pseudorapidity $(\eta)$ of the spectator 
quark ( $\mathrm{q}^{\prime}$ in Fig. 1 (a)) is a characteristic property of the $t$-channel single top production. A significant amount of backgrounds are rejected with the $\left|\eta_{\mathrm{j}^{\prime}}\right|>2.5$ requirement. To further reduce the $t \bar{t}$ background contamination, only positively-charged muons are selected. Figure 3 illustrates the mass of the top quark candidate after selection. The top quark mass distribution in single top and the remaining $t \bar{t}$ events are modeled with Crystal Ball shapes with a difference in their mean value equal to $\Delta m_{\mathrm{t}}=m_{\mathrm{t}}(t$-ch. $)-m_{\mathrm{t}}(t \bar{t})=0.38 \pm 0.17 \mathrm{GeV}$, obtained from simulation. The $\Delta m_{\mathrm{t}}$ value is

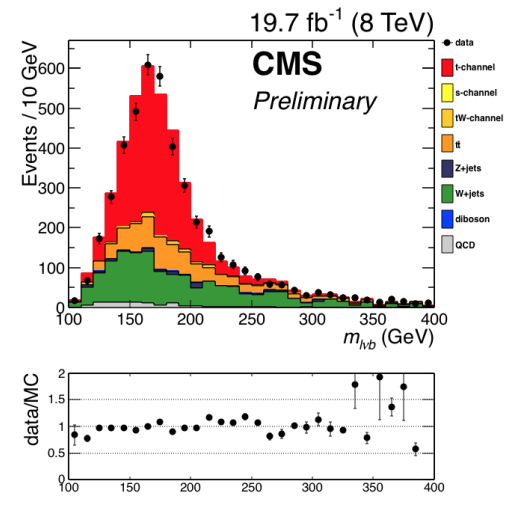

(a)

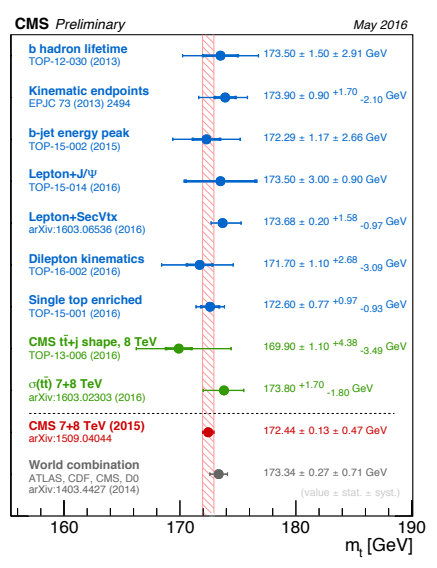

(b)

Figure 3: Reconstructed top quark mass distribution for data (points) and Monte Carlo events (stacked histograms) after the final selection with the bottom panel showing the ratio of the observed number of events in real data to the number predicted by simulation (a). Summary of the CMS top quark mass measurements using methods and properties alternative to the usual mass reconstruction in $\bar{t} \bar{t}$ events (b).

fixed in the fit performed to the top quark mass distribution in the selected sample of reconstructed data events. The measurement yields $m_{\mathrm{t}}=172.60 \pm 0.77$ (stat. ${ }_{-0.93}^{+0.97}$ (syst.) which in terms of precision is better than many of the so-called "alternative" mass measurements in $t \bar{t}$, as presented in Fig. 3 (b).

\section{Top Quark Polarization}

From the theory point of view, the top quark polarization is unambiguously accessible in the $t$-channel single top events. The CMS experiment has measured [8], in $\mu+$ jets final state, the differential $\sigma_{t \text {-ch. }}$ in bins of $\cos \theta_{\mu}^{*}$ where the polarization angle $\theta_{\mu}^{*}$ is defined, in the top quark rest frame, as the angle between the jet corresponding to the spectator quark and the lepton from the top quark decay. The top quark polarization, $P_{\mathrm{t}}$, is extracted from a fit to the unfolded data distribution according to

$$
\frac{1}{\Gamma} \frac{d \Gamma}{d \cos \theta_{\mu}^{*}}=\frac{1}{2}\left(1+P_{\mathrm{t}} \alpha_{\mu} \cos \theta_{\mu}^{*}\right),
$$

where $\alpha_{\mu}$ being the spin analyzing power is close to one for the lepton from the top quark decay. The measurement yields $\frac{1}{2} P_{\mathrm{t}} \alpha_{\mu}=0.26 \pm 0.03$ (stat.) \pm 0.10 (syst.) in agreement, within $2 \sigma$, with the SM expectation 0.44 . 


\section{Search for Top-photon FCNC process}

The CMS experiment has performed a search for the FCNC t $\gamma \mathrm{q}$ interaction in single top events [9]. Because of the difference in parton distribution functions for the incoming $\mathrm{u}$ and $\mathrm{c}$ quarks from the proton, the sensitivity of the search is expected to be different for the $t \gamma \mathrm{c}$ and $t \gamma \mathrm{u}$ vertices. Such distinction is not expected from similar searches in $\bar{t} \bar{t}$ where the FCNC process happens in top quark decays (Fig. 2 (c)). Two different boosted decision tree (BDT) discriminators are built for $\mathrm{t} \gamma \mathrm{u}$ and $\mathrm{t} \gamma \mathrm{c}$ couplings where the one for $\mathrm{t} \gamma \mathrm{u}$ is shown in Fig. 4 (a). The BDT discriminators are fitted to the data, leading to the most stringent limits on $\mathscr{B}(\mathrm{t} \rightarrow \mathrm{c} \gamma)<1.7 \times 10^{-3}$ and $\mathscr{B}(\mathrm{t} \rightarrow$ $\mathrm{u} \gamma)<1.3 \times 10^{-4}$ at $95 \%$ confidence level (CL). Fig. 5 (a) illustrates the CMS results for this search in comparison with results from other experiments.

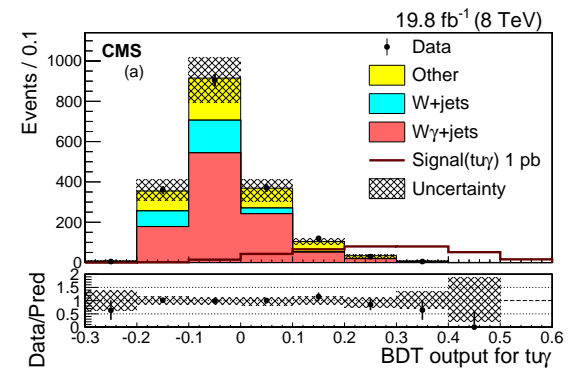

(a)

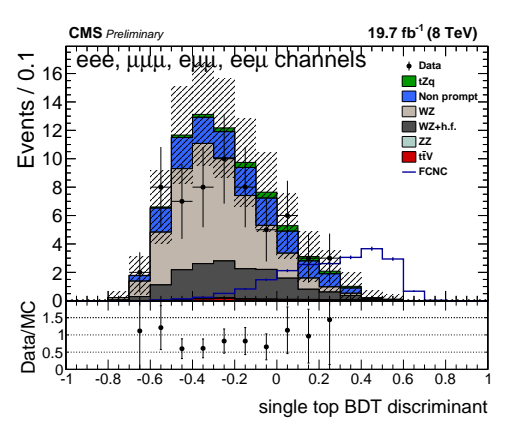

(b)

Figure 4: The BDT output distributions for the data (points), the backgrounds (histograms) after the fit, and the expected $\mathrm{t} \gamma \mathrm{u}(\mathrm{a})$ and $\mathrm{tZu}$ (b) signals (solid lines). The FCNC distribution is normalized to a cross section of $1 \mathrm{pb}$ for (a) where $\mathscr{B}(\mathrm{t} \rightarrow \mathrm{uZ})=0.1 \%$ is assumed for (b). The lower plots show the ratio of the data to the SM prediction.

\section{Search for Top-Z FCNC process}

The search for $\mathrm{tZq}$ FCNC processes in single top events is carried out in the $3 \ell$ final state [10]. The FCNC interaction is considered for the production of the single top quark as well as for the top quark decay in $t \bar{t}$ events. Events in the single top signal region are required to have exactly one jet which is b-tagged. In $\mathrm{t} \overline{\mathrm{t}} \rightarrow \mathrm{WbZq}$ signal region, at least two jets are required with at least one being identified as b-jet. Two BDT discriminators are constructed in the single top and $\bar{t} \overline{\mathrm{t}}$ FCNC regions, sensitive to new physics in the production and decay of the top quark, respectively. A control region with zero b-jet is defined to estimate the contribution of mis-identified leptons as well as the WZ background.

A simultaneous fit is performed to the data in signal regions and in the background control region to set limits on $\mathscr{B}(\mathrm{t} \rightarrow \mathrm{qZ})$ while controlling the contribution of SM backgrounds. The BDT discriminants in signal regions together with the $\mathrm{W}$ boson transverse mass $m_{\mathrm{T}}^{\mathrm{W}}$ in the control region are served as inputs to the fit. The $m_{\mathrm{T}}^{\mathrm{W}}$ template for mis-identified leptons is taken from another control region with the inverted isolation criterion of the lepton that is not from the $\mathrm{Z}$ boson decay. Figure 5 (b) shows the limits where both $\mathrm{tZc}$ and $\mathrm{tZu}$ couplings are allowed to float 
in the fit. Floating one coupling at the time, the analysis yields $\mathscr{B}(\mathrm{t} \rightarrow \mathrm{cZ})<4.9 \times 10^{-4}$ and $\mathscr{B}(\mathrm{t} \rightarrow \mathrm{uZ})<2.2 \times 10^{-4}$ at $95 \% \mathrm{CL}$.

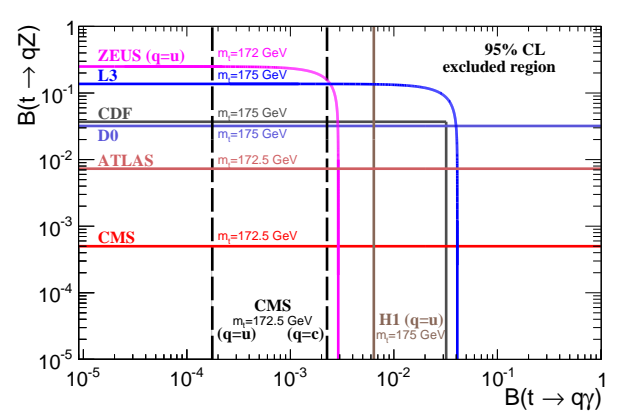

(a)

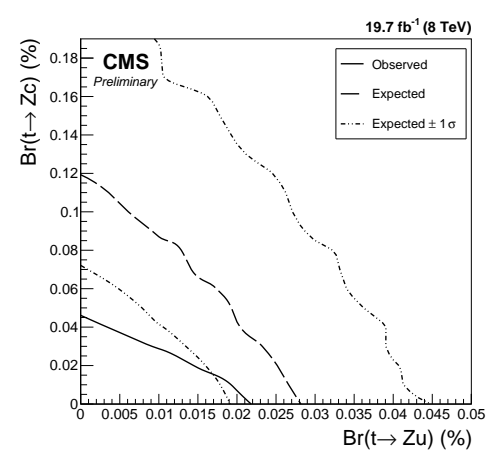

(b)

Figure 5: Comparison of CMS limits on FCNC $\mathscr{B}(\mathrm{t} \rightarrow \mathrm{q} \gamma)$ to those of other experiments (a). Limits on FCNC $\mathscr{B}(\mathrm{t} \rightarrow \mathrm{cZ})$ and $\mathscr{B}(\mathrm{t} \rightarrow \mathrm{uZ})$ extracted simultaneously in from the fit described in the text $(\mathrm{b})$.

\section{Search for SM Z-associated Single-top Production}

For the first time, the search for the SM tZq production has been carried out in CMS [10]. The $3 \ell$ events with at least two jets and at least one b-tagged jet are selected. Compared to the FCNC selection, (Sec.5) a broader $\eta$ range is allowed for jets to increase the signal acceptance (Fig. 2(a)). Similar to Sec. 5, a control region with zero b-tagged jets is used to estimate the contribution of mis-identified leptons and the WZ background. A BDT discriminator in the signal region is trained against the SM t⿱t and $\bar{t} Z$ Z (Fig. 6 (a)). The BDT distribution in signal region and the $m_{\mathrm{T}}^{\mathrm{W}}$ distribution in control region (Fig. $6(\mathrm{~b})$ ) are fitted simultaneously. The cross section in the $3 \ell$ final state is measured to be $\sigma_{\mathrm{tZq}}^{3 \ell}=10_{-7}^{+8} \mathrm{fb}$ with an observed significance of $2.4 \sigma$, in agreement with the SM prediction, $8.2_{-0.03}^{+0.59}$.

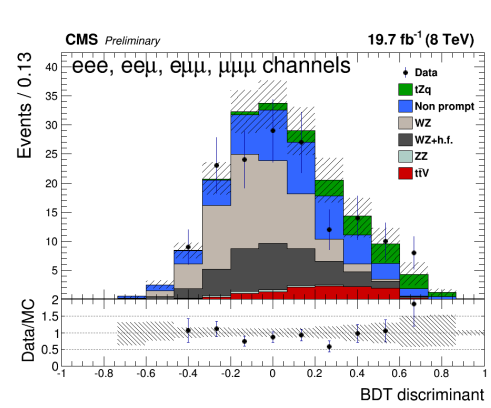

(a)

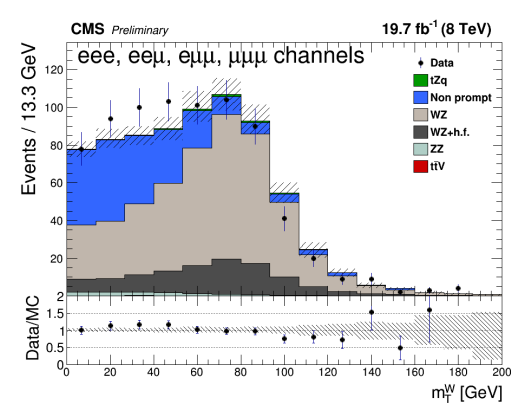

(b)

Figure 6: Data to prediction comparisons after performing the fit for the BDT responses in the signal region (a) and for the $m_{\mathrm{T}}^{\mathrm{W}}$ distribution in the control region (b) for the SM tZq cross section measurement. 


\section{Summary}

The CMS experiment has thoroughly studied top quark properties in single top production. A selected number of results are presented in this note. The measurements and searches are found to have competitive sensitivities to similar analyses using $t \bar{t}$ events. So far no deviation from the standard model is observed although the large data sample expected from the LHC at $13 \mathrm{TeV}$ may change the picture.

\section{Acknowledgments}

The author would like to thank the LHC team and the CMS collaboration at CERN for the excellent work in providing these results. Also thanks to FNRS (Fond National de la Recherche Scientifique) from Belgium who financially supported the author for this conference.

\section{References}

[1] ATLAS Collaboration, Measurement of the inclusive cross-sections of single top-quark and top-antiquark $t$-channel production in pp collisions at $\sqrt{s}=13 \mathrm{TeV}$ with the ATLAS detector, submitted to JHEP, [hep-ex/1609.03920].

[2] CMS Collaboration, Cross section measurement of $t$-channel single top quark production in $p p$ collisions at $\sqrt{s}=13 \mathrm{TeV}$, accepted by Phys. Lett. B, [hep-ex/1610.00678].

[3] ATLAS Collaboration, Measurement of the production cross-section of a single top quark in association with a W boson at 8 TeV with the ATLAS experiment, JHEP 01 (2016) 064, [hep-ex/1510.03752].

[4] CMS Collaboration, Observation of the associated production of a single top quark and a $W$ boson in pp collisions at $\sqrt{s}=8$ TeV, Phys. Rev. Lett. 112 (2014) 231802, [hep-ex/1401.2942].

[5] ATLAS Collaboration, Evidence for single top-quark production in the s-channel in proton-proton collisions at $\sqrt{s}=8 \mathrm{TeV}$ with the ATLAS detector using the Matrix Element Method, Phys. Lett. B 756 (2016) 228, [hep-ex/1511.05980].

[6] CMS Collaboration, Search for s-channel single top quark production in pp collisions at $\sqrt{s}=7$ and 8 TeV, JHEP 09 (2016) 027, [hep-ex/1603.02555].

[7] CMS Collaboration, Measurement of the top quark mass from single-top production events, paper in preparation, [CMS-TOP-PAS-15-001].

[8] CMS Collaboration, Measurement of top quark polarisation in t-channel single top quark production, JHEP 04 (2016) 073, [hep-ex/1511. 02138].

[9] CMS Collaboration, Search for anomalous single top quark production in association with a photon in pp collisions at $\sqrt{s}=8 \mathrm{TeV}$, JHEP 04 (2016) 035, [hep-ex/1511.03951].

[10] CMS Collaboration, Search for associated production of a $Z$ boson with a single top quark and for $\mathrm{tZ}$ flavour-changing interactions in pp collisions at $\sqrt{s}=8 \mathrm{TeV}$, after the conference, the updated version of the paper has been submitted to JHEP, [hep-ex/1702.01404]. 